\title{
L-Lactate Transport and Metabolism in Mitochondria of Hep G2 Cells - The Cori Cycle Revisited
}

\author{
Salvatore Passarella ${ }^{1 *}$ and Avital Schurr ${ }^{2}$ \\ 'School of Medicine University "Aldo Moro", Piazza Giulio Cesare, Bari, Italy, ${ }^{2}$ Department of Anesthesiology and \\ Perioperative Medicine, University of Louisville School of Medicine, Louisville, KY, United States
}

Keywords: L-lactate, L-lactate dehydrogenase, mitochondria, cancer, Cori cycle, mitochondrial transport

In addition to being a glucose precursor in liver and kidney, L-lactate is now also being recognized as an energy substrate in most cells via its oxidation to pyruvate. This oxidation, assumed to occur in the cytosol, is catalyzed by L-lactate dehydrogenase with pyruvate subsequently catabolized in the mitochondria. However, recently mitochondria were recognized to play a role in L-lactate metabolism: the existence of a mitochondrial L-lactate dehydrogenase (m-L-LDH) was suggested by Dianzani (1), and later demonstrated by Baba and Sharma (2) to be located in the mitochondrial matrix (3). Indeed, L-lactate transport and metabolism was shown in various mitochondria, including skeletal muscle (4) rat heart (5), liver (6), brain (7-9), cerebellar granule cells (10), rabbit gastrocnemius (11), sperm cells (12), pig liver (13), and even plant (14). Thus, the existence of m-L-LDH, as reviewed by

OPEN ACCESS

Edited by:

Sergio Giannattasio,

Istituto di Biomembrane,

Bioenergetica e Biotecnologie

Molecolari (IBIOM), Italy

Reviewed by:

Giuseppe Paradies,

Università degli studi di Bari Aldo

Moro, Italy

*Correspondence:

Salvatore Passarella spassarella3@gmail.com

Specialty section:

This article was submitted to Molecular and Cellular Oncology,

a section of the journal

Frontiers in Oncology

Received: 12 March 2018

Accepted: 04 April 2018

Published: 23 April 2018

Citation:

Passarella S and Schurr A (2018)

L-Lactate Transport and Metabolism in Mitochondria of Hep G2 Cells-

The Cori Cycle Revisited.

Front. Oncol. 8:120.

doi: 10.3389/fonc.2018.00120 Passarella et al. (3), Brooks (15), and Schurr (16), was recognized with its inclusion in the MitoCarta (http://www.broadinstitute.org/pubs/MitoCarta/index.htrnl). As expected, in light of the presence of the L-LDH in the matrix, the occurrence of carriers for L-lactate has been shown in functional studies with purified, coupled mitochondria. These include the L-lactate $/ \mathrm{H}^{+}$symporter and the L-lactate/ pyruvate and L-lactate/oxaloacetate antiporters (3). Surprisingly, the overwhelming evidence for an $\mathrm{m}-\mathrm{L}-\mathrm{LDH}$ located inside mitochondria is not universally accepted, with some scientists still being skeptic about the existence of $\mathrm{m}$-L-LDH, while others localizing $\mathrm{m}-\mathrm{L}-\mathrm{LDH}$ in the intermembrane space (17). It is our opinion that the skepticism could originate due to difficulties in isolating coupled mitochondria, not an easy task, in particular with skeletal muscle samples, or not being careful enough in selecting reaction media and in using inhibitors at the correct concentration (11). That $\mathrm{m}$-L-LDH is localized inside mitochondria will be shown below.

\section{IS L-LACTATE BEING TRANSPORTED AND METABOLIZED IN CANCER CELL MITOCHONDRIA?}

Yes, it is. Although in the 1920s, Warburg found that cancer cells prefer to produce ATP by glycolysis with L-lactate production, to the best of our knowledge, the mitochondrial metabolism of L-lactate had not been investigated in cancer cells until 2010, when the first evidence for L-lactate mitochondrial metabolism in these cells (already reported in 2008 by Gabriella Chieppa in her PhD thesis at the University of Molise) was published (18). In this case, to study L-lactate transport and metabolism in mitochondria isolated from both normal and cancer prostate cells, spectroscopic and polarographic techniques were used, in which either m-L-LDH reaction or oxygen consumption by mitochondria, supplied with externally added L-lactate were monitored, respectively (19), rather than employing more involved procedures, available in molecular biology, genetics, and chemistry laboratories. The former two techniques were chosen since they afford the continuous monitoring of the kinetics of the investigated processes in experiments that last for several minutes where mitochondria remain coupled. By contrast, measurements using the latter methods are usually made once the processes have already been completed. Accordingly, an increase in the redox state of the 
intramitochondrial pyridine nucleotides, as shown by fluorimetric measurements, upon the addition of L-lactate to mitochondria indicates that L-lactate metabolism occurs inside the organelles via an $\mathrm{NAD}^{+}$-dependent $\mathrm{m}$-L-LDH; unfortunately, the occurrence of the mitochondrial L-lactate metabolism in cancer cells was not quoted in Ferguson et al. (17) possibly because the authors of the review consider the spectroscopic and polarographic techniques to be "problematic," despite its widespread use by numerous scientists. That theirs is a minority opinion might be exemplified by quoting from a review by Mayevsky and Rogatsky (20), which states that "The large numbers of publications by different groups testify to the valuable information gathered in various experimental conditions. The monitoring of NADH levels in the tissue provides the most important information on the metabolic state of the mitochondria." The existence of $\mathrm{m}-\mathrm{L}-\mathrm{LDH}$ can be also immunologically confirmed in mitochondria that are proven to be free of cytosolic contamination.

Notice that in the case where $\mathrm{m}-\mathrm{L}-\mathrm{LDH}$ is proposed to be localized in the intermembrane space, the increase in the intramitochondrial pyridine nucleotide fluorescence is explained as follows: L-lactate enters the mitochondrial intermembrane space where it is oxidized to pyruvate, which in turn crosses the mitochondrial inner membrane to be oxidized inside the mitochondria via the pyruvate dehydrogenase complex [for review, see Ferguson et al. (17)]. Such a mechanism is not supported by various experimental findings. For instance, in de Bari et al. (18), it was shown that $\mathrm{NAD}^{+}$reduction proceeds despite the presence of arsenite, an inhibitor of pyruvate dehydrogenase, but is inhibited by oxamate, an inhibitor of L-LDH. Additional evidence against the presence of $\mathrm{m}-\mathrm{L}-\mathrm{LDH}$ in the intermembrane space emerges from experimental results showing that L-lactate enters mitochondria under conditions where pyruvate is a non-penetrant compound (21) or where the pyruvate/ $\mathrm{H}^{+}$symporter is blocked by an inhibitor (6). These experimental approaches can be also applied to measurements of oxygen consumption (in the presence or absence of ADP), proton efflux and membrane potential generation in the future. By applying the control strength criterion with various non-penetrant inhibitors (19) it can be established whether or not the rate of the above processes mirrors that of L-lactate transport across the mitochondrial membrane. Thus, L-lactate transport can be investigated quantitatively, including the occurrence of hyperbolic kinetics, $\mathrm{pH}$ profile, etc. Moreover, comparison made between the inhibition profiles of pyruvate and L-lactate-dependent mitochondrial processes through the use of compounds that are unable to enter mitochondria allows for a distinction between L-lactate and pyruvate carriers.

Briefly, it has also been shown that externally added L-lactate can enter both normal and cancer prostate cells and in particular, in a carrier-mediated manner, enters their mitochondria, where an $\mathrm{L}-\mathrm{LDH}$ exists and is located in the inner compartment. The $\mathrm{m}$-L-LDHs have been demonstrated to differ from the cytosolic enzymes that themselves differ from one another. Normal and cancer cells show differences with respect to $\mathrm{m}-\mathrm{L}-\mathrm{LDH}$ protein level and activity, where both the enzyme expression and activity are higher in cancer cells.

In 2011, the existence of monocarboxylate transporter (MCT) and $\mathrm{LDH}$ proteins in mitochondrial reticula of breast cancer cell lines was demonstrated (22). In that case, the expression of both MCTs and L-LDH was measured, and their mitochondrial localization was determined via immunofluorescence, a technique that does not allow for the identification of the submitochondrial localization.

A broader investigation of L-lactate transport and metabolism in cancer cell mitochondria was carried out in human hepatocellular carcinoma (Hep G2) cells (21) in which gluconeogenesis takes place (23). Hep G2 cell mitochondria (Hep G2-M) possess an $\mathrm{m}-\mathrm{L}-\mathrm{LDH}$ restricted to the inner mitochondrial compartment. Cytosolic and mitochondrial L-LDHs were also found to differ from one another in their saturation kinetics. The occurrence of a carrier-mediated L-lactate transport in these mitochondria has also been shown. Importantly, the efflux of various metabolites, including pyruvate, oxaloacetate, malate, and citrate, resulting from L-lactate addition to mitochondria was first shown, this giving a first insight into the role of mitochondrial metabolism of L-lactate; accordingly, the occurrence of an L-lactate/pyruvate shuttle devoted to the oxidation of the cytosolic NADH was also shown. Ultimately, the removal of the oxidation product by carrier-mediated transport and mitochondrial metabolism overcomes any theoretical thermodynamic difficulty which was considered to rule out any L-lactate oxidation in the mitochondria.

These findings strongly suggest that a revision of the dogmatic view of glucose metabolism is needed with a special focus on the role of L-lactate and $\mathrm{m}-\mathrm{L}-\mathrm{LDH}$ in gluconeogenesis. Hence, the Cori cycle (formulated in 1929 as an energy-requiring metabolic pathway in animals, where carbon atoms of glucose pass along the circular route: muscle glycogen $\rightarrow$ blood lactate $\rightarrow$ liver (where gluconeogenesis occurs) $\rightarrow$ blood glucose $\rightarrow$ muscle glucose $\rightarrow$ muscle glycogen) demands revision, too. In this regard, cellular L-lactate oxidation, which is necessary for the production of glucose in the Cori cycle, has been traditionally postulated to take place in the cytosol, but is it? The cytosolic$\mathrm{L}-\mathrm{LDH}(\mathrm{c}-\mathrm{L}-\mathrm{LDH})$ is a reducing enzyme, the final step of the glycolytic pathway, which converts pyruvate to L-lactate, and thus provides the regeneration of $\mathrm{NAD}^{+}$. This reaction should proceed unabated, independently of the presence or absence of oxygen, as the standard free-energy $\left(\Delta G^{0^{\prime}}\right)$ change of pyruvate conversion to L-lactate is about $-6 \mathrm{kcal} / \mathrm{mol}$. In addition, the high affinity of pyruvate to $\mathrm{C}-\mathrm{L}-\mathrm{LDH}$ would explain the fact that the normal [L-lactate]/[pyruvate] ratio in blood and other tissues is $>10$, a value that cannot correspond with the proposal of pyruvate as the end product of glycolysis under normal conditions. Therefore, the dogmatic portrayal of this reaction as bidirectional is misleading and has been accepted to date due to the absence of a possible alternative. We contend that L-lactate oxidation back to pyruvate does not take place in the cytosol, but rather, it occurs in the mitochondria. Indeed, there are only two options to prevent L-lactate accumulation in the cytosol, either L-lactate is transported out of the cell (under anaerobic conditions) and/or is oxidized via m-L-LDH upon its transport into the mitochondrion (under aerobic conditions). Therefore, even if we agree with $\mathrm{Lu}$ et al. (24) that "the majority of glycolysisderived pyruvate is diverted to lactate fermentation," we cannot accept that L-lactate is "kept away from mitochondrial oxidative metabolism." 


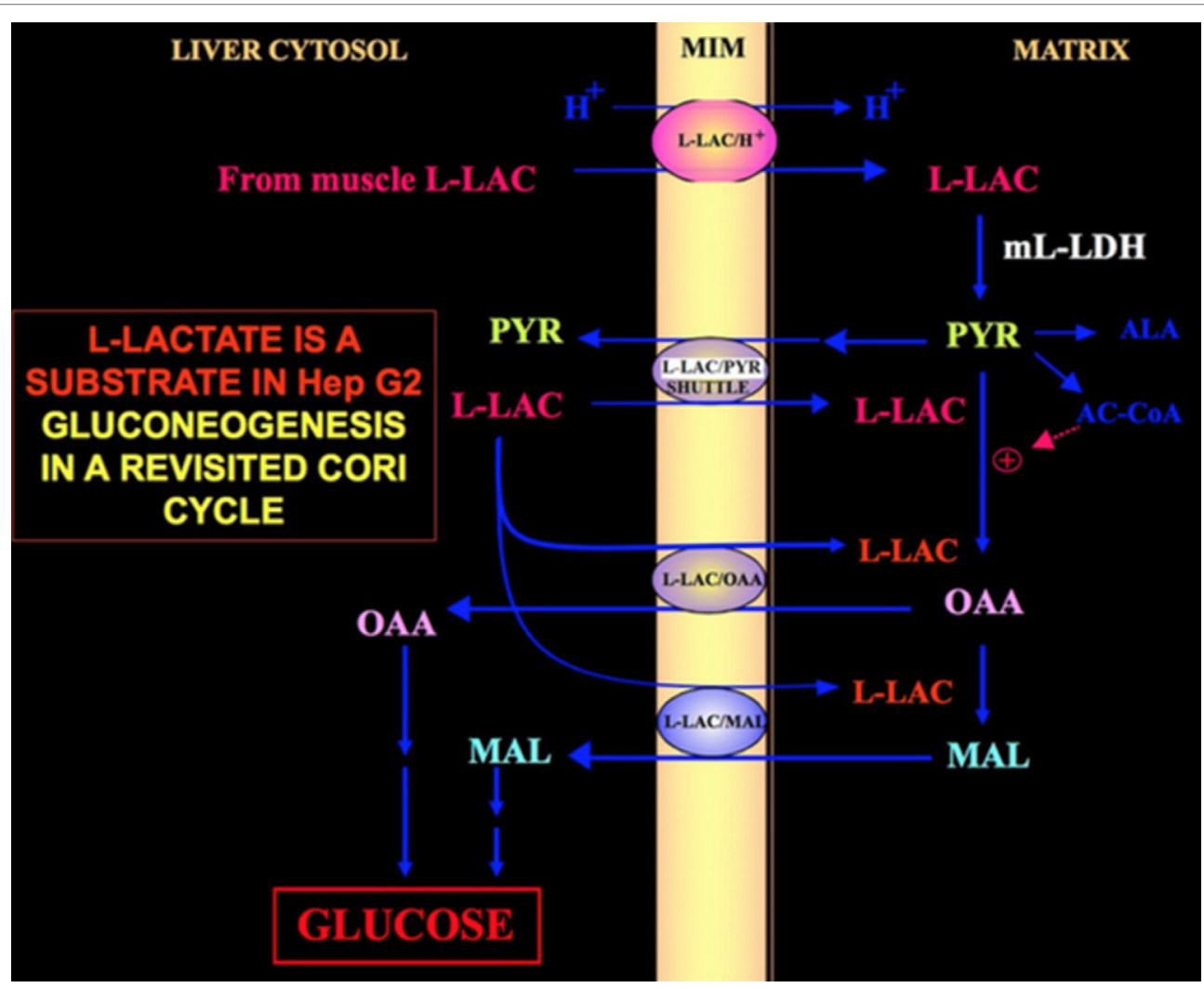

FIGURE 1 | Cori cycle revisited in Hep G2 cells. Given that pyruvate cannot enter Hep G2-M, as shown in Pizzuto et al. (21), L-lactate produced in the muscles reaches the liver via the blood stream and from the cytosol enters mitochondria; in the matrix L-lactate metabolism gives rise to pyruvate (PYR) via m-L-LDH and then to oxaloacetate (OAA) and malate (MAL) that are exported from the mitochondria to the cytosol via three putative carriers to be used for the I-lactate pyruvate shuttle and for gluconeogenesis to occur via a mechanism similar to that already shown by de Bari et al. (6).

Of special interest is the fact that pyruvate cannot enter Hep G2-M. In fact, contrary to malate + glutamate and L-lactate, externally added pyruvate fails to cause either oxygen consumption or membrane potential generation [see Pizzuto et al. (21) for details]. Notice that an impairment of pyruvate transport in cancer cells has been reported by Paradies et al. (25). Therefore, independently of the theoretical unfeasibility of L-lactate oxidation in the cytosol, as was explained above, the classic Cori cycle cannot occur in Hep G2cells. Therefore, we offer a revised Cori cycle (Figure 1), which involves both the mitochondrial carriers that mediate the L-lactate-dependent traffic and the m-L-LDH, which provides pyruvate inside mitochondria. Accordingly, the appearance outside mitochondria of oxaloacetate and malate derived from L-lactate uptake and metabolism via m-L-LDH, pyruvate dehydrogenase, pyruvate carboxylase, and malate dehydrogenase and by exchanges, likely due to the L-lactate/ oxaloacetate and L-lactate/malate antiporters, confirms an anaplerotic role for L-lactate in gluconeogenesis in which mitochondria play a unique role. Importantly, the addition of L-lactate to Hep G2-M results in the appearance outside mitochondria of citrate, the fatty acid precursor. Accordingly, by using high-resolution mass spectrometry, L-lactate uptake into mitochondria of HeLa and H460 cells was found and proved to result in lipid synthesis; additionally, transmission electron microscopy confirmed that $\mathrm{LDH}$ is localized to the mitochondria (26). Surprisingly, the anaplerotic role of L-lactate mitochondrial metabolism has not been considered when cancer metabolism was "reexamined" (27).

We believe that the proposed revision of the Cori cycle, necessary for Hep G2 cells, should also be considered in all other types of cells where mitochondrial metabolism of L-lactate is active. For instance, partial reconstruction of in vitro gluconeogenesis arising from mitochondrial L-lactate uptake/metabolism was shown in the absence of LDH outside mitochondria (6).

The role of the mitochondrial L-lactate metabolism merits further focus: given that hydrogen peroxide production in the tumor microenvironment fuels the anabolic growth of cancer cells (28), a possible role of the putative mitochondrial L-lactate oxidase (LOX) which generates hydrogen peroxide in rat liver mitochondria (29) should be investigated; the LOX existence in Hep G2-M appears to be consistent with the evidence that rotenone, which blocks oxygen consumption induced by the addition of malate + glutamate fails to inhibit oxygen consumption induced by the addition of L-lactate.

\section{AUTHOR CONTRIBUTIONS}

SP conceived this opinion, shared it and wrote the paper with AS. 


\section{REFERENCES}

1. Dianzani MU. Distribution of lactic acid oxidase in liver and kidney cells of normal rats and rats with fatty degeneration of the liver. Arch Fisiol (1951) 50:181-6.

2. Baba N, Sharma HM. Histochemistry of lactic dehydrogenase in heart and pectoralis muscles of rat J Cell Biol (1971) 51:621-35.

3. Passarella S, de Bari L, Valenti D, Pizzuto R, Paventi G, Atlante A. Mitochondria and L-lactate metabolism. FEBS Lett (2008) 582:3569-76. doi:10.1016/j. febslet.2008.09.042

4. Dubouchaud H, Butterfield GE, Wolfel EE, Bergman BC, Brooks GA. Endurance training, expression, and physiology of LDH, MCT1, and MCT4 in human skeletal muscle. Am J Physiol Endocrinol Metab (2000) 278:E571-9. doi:10.1152/ajpendo.2000.278.4.E571

5. Valenti D, de Bari L, Atlante A, Passarella S. L-lactate transport into rat heart mitochondria and reconstruction of the L-lactate/pyruvate shuttle. Biochem J (2002) 15:101-4. doi:10.1042/bj3640101

6. de Bari L, Atlante A, Valenti D, Passarella S. Partial reconstruction of in vitro gluconeogenesis arising from mitochondrial L-lactate uptake/metabolism and oxaloacetate export via novel L-lactate translocators. Biochem J (2004) 380:231-42. doi:10.1042/bj20031981

7. Schurr A. Lactate: the ultimate cerebral oxidative energy substrate? J Cereb Blood Flow Metab (2006) 26:142-52. doi:10.1038/sj.jcbfm.9600174

8. Schurr A, Payne RS. Lactate, not pyruvate, is neuronal aerobic glycolysis end product: an in vitro electrophysiological study. Neuroscience (2007) 147:613-9. doi:10.1016/j.neuroscience.2007.05.002

9. Hashimoto T, Hussien R, Cho H-S, Kaufer D, Brooks GA. Evidence for a mitochondrial lactate oxidation complex in rat neurons: a crucial component for a brain lactate shuttle. PLoS One (2008) 3:e2915. doi:10.1371/journal. pone.0002915

10. Atlante A, de Bari L, Bobba A, Marra E, Passarella S. Transport and metabolism of L-lactate occur in mitochondria from cerebellar granule cells and are modified in cells undergoing low potassium dependent apoptosis. Biochim Biophys Acta (2007) 1767:1285-99. doi:10.1016/i.bbabio.2007.08.003

11. Passarella S, Paventi G, Pizzuto R. The mitochondrial L-lactate dehydrogenase affair. Front Neurosci (2014) 8:407. doi:10.3389/fnins.2014.00407

12. Paventi G, Lessard C, Bailey JL, Passarella S. In boar sperm capacitation L-lactate and succinate, but not pyruvate and citrate, contribute to the mitochondrial membrane potential increase as monitored via safranine $\mathrm{O}$ fluorescence. Biochem Biophys Res Commun (2015) 462:257-62. doi:10.1016/j. bbrc.2015.04.128

13. Paventi G, Pizzuto R, Passarella S. The occurrence of L-lactate dehydrogenase in the inner mitochondrial compartment of pig liver. Biochem Biophys Res Commun (2017) 489:255-61. doi:10.1016/j.bbrc.2017.05.154

14. Paventi G, Pizzuto R, Chieppa G, Passarella S. L-lactate metabolism in potato tuber mitochondria. FEBS J (2007) 274:1459-69. doi:10.1111/j. 1742-4658.2007.05687.x

15. Brooks GA. Cell-cell and intracellular lactate shuttles. J Physiol (2009) 587:5591-600. doi:10.1113/jphysiol.2009.178350

16. Schurr A. Cerebral glycolysis: a century of persistent misunderstanding and misconception. Front Neurosci (2014) 8:360. doi:10.3389/fnins.2014. 00360

17. Ferguson BS, Rogatzki MJ, Goodwin ML, Kane DA, Rightmire Z, Gladden LB. Lactate metabolism: historical context, prior misinterpretations, and current understanding. Eur J Appl Physiol (2018) 118:691-728. doi:10.1007/s00421-017-3795-6
18. de Bari L, Chieppa G, Marra E, Passarella S. L-lactate metabolism can occur in normal and cancer prostate cells via the novel mitochondrial L-lactate dehydrogenase. Int J Oncol (2010) 37:1607-20. doi:10.3892/ijo-00000815

19. Passarella S, Atlante A, Valenti D, de Bari L. The role of mitochondrial transport in energy metabolism. Mitochondrion (2003) 2:319-43. doi:10.1016/ S1567-7249(03)00008-4

20. Mayevsky A, Rogatsky GG. Mitochondrial function in vivo evaluated by NADH fluorescence: from animal models to human studies. Am J Physiol Cell Physiol (2007) 292:C615-40. doi:10.1152/ajpcell.00249.2006

21. Pizzuto R, Paventi G, Porcile C, Sarnataro D, Daniele A, Passarella S. L-lactate metabolism in HEP G2 cell mitochondria due to the L-lactate dehydrogenase determines the occurrence of the lactate/pyruvate shuttle and the appearance of oxaloacetate, malate and citrate outside mitochondria. Biochim Biophys Acta (2012) 1817:1679-90. doi:10.1016/j.bbabio.2012.05.010

22. Hussien R, Brooks GA. Mitochondrial and plasma membrane lactate transporter and lactate dehydrogenase isoform expression in breast cancer cell lines. Physiol Genomics (2011) 43:255-64. doi:10.1152/physiolgenomics. 00177.2010

23. Okamoto T, Kanemoto N, Ban T, Sudo T, Nagano K, Niki I. Establishment and characterization of a novel method for evaluating gluconeogenesis using hepatic cell lines, H4IIE and HepG2. Arch Biochem Biophys (2009) 491:46-52. doi:10.1016/j.abb.2009.09.015

24. Lu J, Tan M, Cai Q. The Warburg effect in tumor progression: mitochondrial oxidative metabolism as an anti-metastasis mechanism. Cancer Lett (2015) 356:156-64. doi:10.1016/j.canlet.2014.04.001

25. Paradies G, Capuano F, Palombini G, Galeotti T, Papa S. Transport of pyruvate in mitochondria from different tumor cells. Cancer Res (1983) 43:5068-71.

26. Chen YJ, Mahieu NG, Huang X, Singh M, Crawford PA, Johnson SL, et al. Lactate metabolism is associated with mammalian mitochondria. Nat Chem Biol (2016) 12:937-43. doi:10.1038/nchembio.2172

27. San-Millán I, Brooks GA. Reexamining cancer metabolism: lactate production for carcinogenesis could be the purpose and explanation of the Warburg effect. Carcinogenesis (2017) 38:119-33. doi:10.1093/carcin/bgw127

28. Martinez-Outschoorn UE, Lin Z, Trimmer C, Flomenberg N, Wang C, Pavlides S, et al. Cancer cells metabolically "fertilize" the tumor microenvironment with hydrogen peroxide, driving the Warburg effect: implications for PET imaging of human tumors. Cell Cycle (2011) 10:2504-20. doi:10.4161/ cc.10.15.16585

29. de Bari L, Valenti D, Atlante A, Passarella S. L-lactate generates hydrogen peroxide in purified rat liver mitochondria due to the putative L-lactate oxidase localized in the intermembrane space. FEBS Lett (2010) 584:2285-90. doi:10.1016/j.febslet.2010.03.038

Conflict of Interest Statement: The authors declare that the research was conducted in the absence of any commercial or financial relationships that could be construed as a potential conflict of interest.

The reviewer [GP] declared a shared affiliation, with no collaboration, with one of the authors $[\mathrm{SP}]$ to the handling Editor.

Copyright (c) 2018 Passarella and Schurr. This is an open-access article distributed under the terms of the Creative Commons Attribution License (CC BY). The use, distribution or reproduction in other forums is permitted, provided the original author(s) and the copyright owner are credited and that the original publication in this journal is cited, in accordance with accepted academic practice. No use, distribution or reproduction is permitted which does not comply with these terms. 